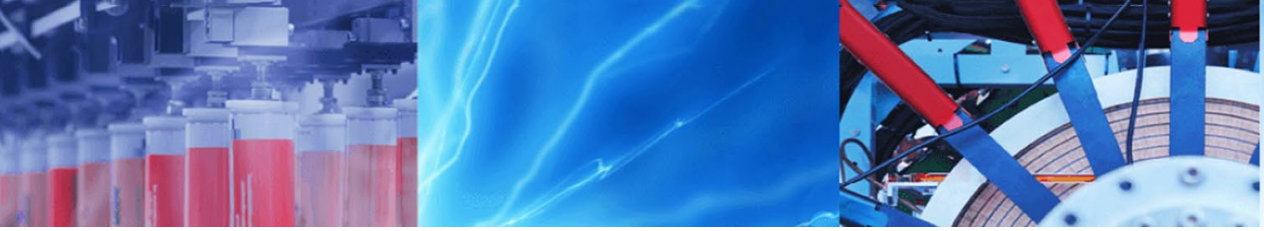

Research Article

\title{
Design and exploration of functioning of a D-Z shaped SNG multiband metamaterial for L-, S-, and X-bands applications
}

\author{
Nipa Dhar ${ }^{1}$ ] Muhammad Asad Rahman ${ }^{1}$ (D) Md. Azad Hossain ${ }^{1}$
}

Received: 1 October 2019 / Accepted: 3 May 2020 / Published online: 15 May 2020

(c) Springer Nature Switzerland AG 2020

\begin{abstract}
A design and performance analysis of a new triple-band metamaterial (MTM) is imparted in this article. The proposed MTM is able to produce three operating frequency bands and can be applicable for $\mathrm{L}-, \mathrm{S}-$, and $\mathrm{X}$-bands applications. The $\mathrm{L}$-band and S-band frequency responses are caused by electric resonance, and the $\mathrm{X}$-band is generated by magnetic resonance. The triple-band response is produced by combining two open D-shaped loops coupled with a Z-shaped metal strip. To conclude the electromagnetic behaviour of the proposed MTM, the Nicolson Rose Weir method is utilized, and effective parameter extraction from reflection and transmission coefficient is done. Then the parametric analysis is done by changing the arm length of the loop, varying the capacitive gaps of the structure to analyse the performance of the proposed structure. Moreover, the equivalent circuit analysis of three individual frequency bands is performed for a clear understanding of the resonance phenomena of the proposed MTM. Besides, the analysis of $1 \times 2,2 \times 1,2 \times 2$, and $4 \times 4$ array structures of the unit cells is done to validate the activities of the MTM because array structure is often needed to improve the performance of an antenna. All the structures reveal their triple-band operation and promising for L-, $\mathrm{S}-$, and X-bands applications. The proposed MTM unit cell exhibits a larger effective medium ratio (17.7) for the lower frequency band. So, the projected compact MTM is a promising solution for different microwave applications.
\end{abstract}

Keywords Single negative MTM · Effective medium ratio $\cdot$ Multiband $\cdot$ Unit cell $\cdot$ MTM array

\section{Introduction}

The artificial compound materials that are termed metamaterials (MTMs), are intended to display extraordinary electromagnetic (EM) marvels not accessible by regular materials. The unavailable properties of MTMs such as negative permittivity, chirality, negative permeability, phase engineering, negative refracting index, beam steering, magnetism, polarization conversion, Doppler effects, Cherenkov radiation, Snell's law, etc. make them affluent to offer many fascinating ways of handling light. As a result of these surprising properties, it can be utilized in numerous incredible applications, for example, absorber design [1],
EM absorption reduction [2], invisibility cloaking [3], filter design $[4,5]$, antenna design $[6,7]$, etc.

The MTMs are classified based on their negative values of permittivity and permeability. Single negative MTMs (SNG) have negative values of either permittivity or permeability and well known as $\varepsilon$-negative MTM (ENG) (where permittivity is negative) or $\mu$ - negative MTM (MNG) (where permeability is negative). In a double negative material (DNG), both $\mu$ and $\varepsilon$ are negative.

The electrodynamics of materials having simultaneous negative permeability and permittivity was first observed by Victor Veselago in 1968 [8]. Because of the absence of such natural material, Veselago's invention was unable to draw attention until 1999. A breakthrough occurred in

Nipa Dhar, nipa@cuet.ac.bd; Muhammad Asad Rahman, asad31@cuet.ac.bd; Md. Azad Hossain, azad@cuet.ac.bd|'Faculty of Electrical and Computer Engineering, Chittagong University of Engineering and Technology, Chattogram 4349, Bangladesh. 
2000 when Smith proved a composite medium named left-handed MTM (LHM) with unattainable properties of negative permeability and permittivity [9]. The first physically realized MTM was composed of subwavelength splitring resonators to get effective negative permeability and metallic wire to get negative permittivity $[10,11]$. The concept of a single-negative transmission line (SNG-TL) and complementary right-left handed transmission line first introduced in literature [12] and also the L-C equivalent circuits left-handed properties. For any specific application bands, the size of the MTM unit cells is much smaller than its wavelength. That is why MTMs are attractive to design a miniaturized antenna of multiband applications.

By combining ENG and MNG material in the same unit cell, negative refractive indexed material can be realized [13]. Moreover, SNG materials have been used to create left-handed (LH) material. The applications of MTMs are significant in antenna gain enhancement [14-16], bandwidth enhancement [17], multiband antennas [18-21], and in microwave imaging [22]. In [23], a circularly polarized (CP) zeroth-order negative epsilon MTM has been reported where the asymmetrical unit cells are used to create circular polarization. In many microwave applications, such as multiband operations, gain enhancement, sensor applications, etc. requires multiband frequency operation of MTMs. To find the solution of this situation, different multiband SNG and DNG MTM unit cells can be used and discussed in the literature [24-26]. An open delta-shaped loop within the square resonator having triple-band ENG MTM characteristics is described in [24] where the effective medium ratio is larger in three distinct electric resonance. A dual-band double G-shaped DNG MTM has been realized for C-and S-bands applications in [25]. This dual-band resonator is able to produce one single negative and another DNG operating band. A modified Z-shaped MTM has been investigated in [26] which produces DNG behaviour over a wide frequency band. $A 1 \times 1,2 \times 2$, and $4 \times 4$ arrays of the unit cells with 0 -degree and 90 -degree rotation angles have been analyzed to observe the behaviour of the cell. All of these structure shows DNG operating bands over a wideband. An altered hexagonal shape meta atom for quad-band applications has been reported in [27] for sensor applications. Normal and parallel incidence of EM wave is investigated in this literature. By changing the air height between two unit cells, electric and magnetic resonance can be altered. This scheme is utilized to develop the idea of a pressure sensor. A double arrow structured meta-atom has been reported in [28] suitable for satellite applications and remote correspondence. An $\mathrm{H}$-shaped and a split $\mathrm{H}$-shaped DNG MTM reported in the literature $[29,30]$ for multiband wireless applications.

Unlike conventional ENG MTM having a plasmonic structure [10], recent ENG cells do not require any wire structure. Because wire configurations require a regular connection between unit cells called intercell connection and causes fabrication difficulty. It arises a problem when to design a contoured substance like a curved lens. The wire structure acts as a bulk medium, but researchers facing challenges to accommodate its length within the unit cell. As found, the length of the wire is larger than the unit cells area. To solve these problems, electric field coupledresonator reported in [31]. This nomenclature introduced epsilon negative single loop resonator with a capacitive gap in the middle of the loop forming an electric inductive-capacitive (ELC) circuit and do no need for a continuous path of current flow between unit cells. To reduce the overall size of the unit cell, modification of ELC resonator has been reported in [32]. Increasing total capacitance by fitting an array of the integral capacitor causes a decrease in resonant frequency. This entails an overall size reduction of unit cells compare to its operating wavelength. Further size reduction of electrically coupled resonators has been accomplished by integrating an array of minder lines which violates the capacitive coupling between two resonator rings [33]. A Z-shaped miniaturized ENG MTM has been reported in [34]. This investigation offers a low cost, small-sized meta-atom for high-frequency planar technology. It is able to exhibit lower resonance compared to an ELC resonator for the same physical size, in response to its normal incidence of EM wave. The literature [35] represents a comparison of epsilon negative ELC resonators based on their effective medium ratio. Size reduction is accomplished by introducing multiple metal strips for increasing the value of inductance and integrated capacitor for a higher value of capacitance. To overcome the Bloch mode of higher-order and cross cell interconnection effect, literature [36] reveals a new design composed of a coupled ELC resonators and a loop resonator (hybrid) on the other side of the substrate. This design also offers a higher effective medium ratio compared to conventional designs. A delta loop loaded minder line integrated ENG MTM has been explored in [37] which claims a small structured cell with dual-band capability. A triple-band Greek-Key pattern ENG MTM has been designed and fabricated in [38]. This design provides multiband operation with its compact size. A prototype of a $10 \times 10$ array is fabricated and confirmed its ENG behaviour in distinct bands. A double loop meta-atom has been designed and fabricated on gallium arsenide having a dual-band frequency response in [39]. An MTM revealed by literature [40] where two delta loop resonators and a thin wire is used to develop an SNG and a DNG MTMs. A dual-band butterfly-pattern MTM has been reported in [41], and the two bands are produced due to electric and magnetic resonance, respectively. An M-band nested unconnected $\mathrm{U}$-shaped resonator having $\mathrm{LC}$ resonance in three distinct 


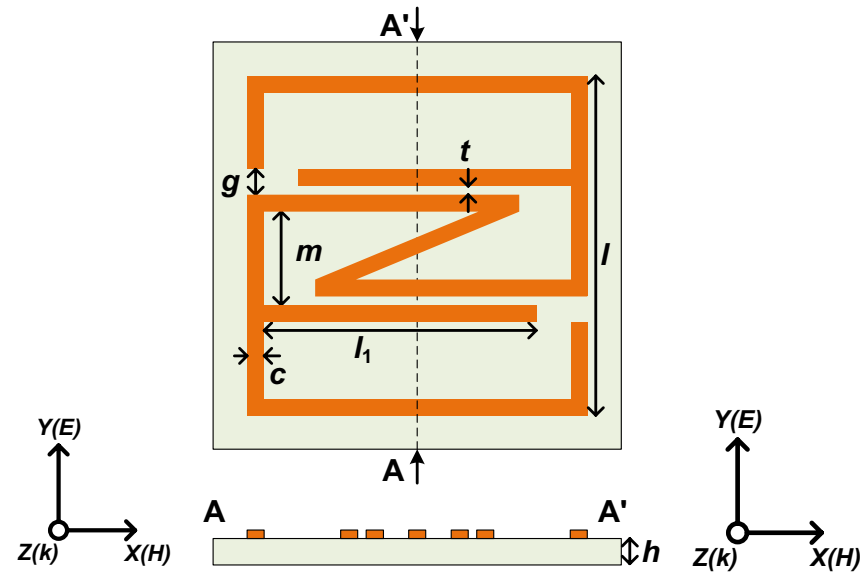

(a)

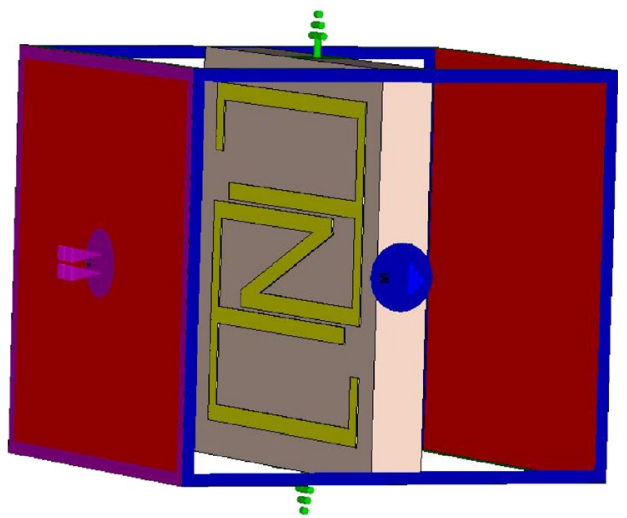

(b)

Fig. 1 Geometry of the proposed triple-band MTM unit cell a Schematic layout and cross-sectional view (AA'), b Simulation setup

Table 1 Dimensions of the proposed MTM unit cell for optimum performance

\begin{tabular}{ll}
\hline Parameters & $\begin{array}{l}\text { Dimen- } \\
\text { sions } \\
(\mathrm{mm})\end{array}$ \\
\hline$l$ & 10 \\
$I_{1}$ & 7.53 \\
$g$ & 1.2 \\
$\mathrm{t}$ & 0.4 \\
$\mathrm{c}$ & 0.5 \\
$\mathrm{~m}$ & 3.2 \\
$h$ & 1.6 \\
\hline
\end{tabular}

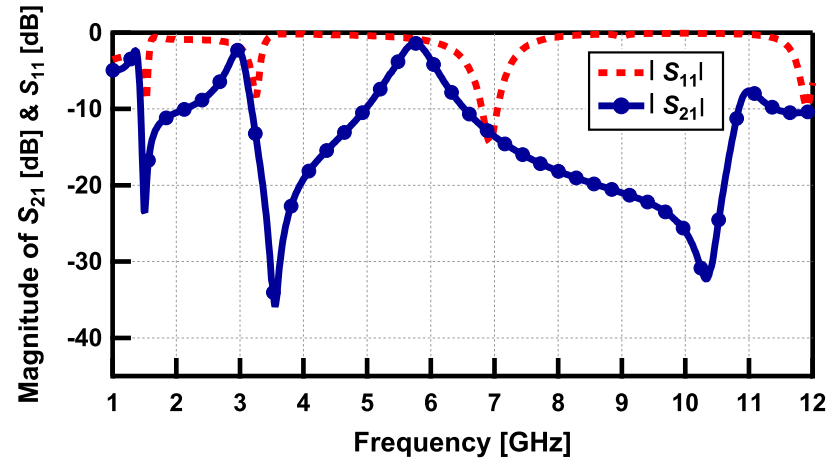

Fig. 2 Magnitude of the transmission $\left(S_{21}\right)$ and reflection coefficient $\left(S_{11}\right)$ curves

operating bands can produce strong magnetic resonance. The resonant frequency can be individually modified by regulating the related U-shaped rings arm length [42]. A near-zero refractive index MTM has been reported in [43] where a pi-shaped structure is used. This design is intended for the application of an X-band cloak operation

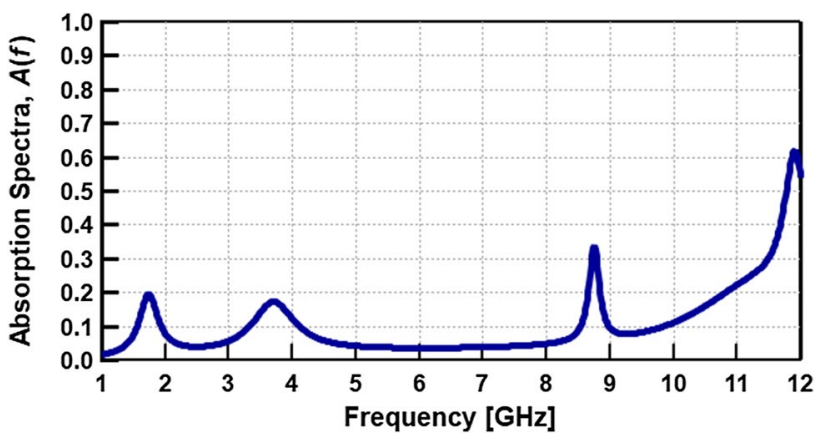

Fig. 3 Absorptivity curve of the proposed triple-band MTM

having an effective medium ratio $\left(\lambda_{0} / a\right)$ of 3.58 . A $22 \times 22$ $\mathrm{mm}^{2}$ epsilon negative unit cell has been designed for C-band filtering application and reported in [44] having an effective medium ratio of 9.01. A reconfigurable antenna is composed of a circular-shaped reconfigurable ENG MTM where capacitive gaps are switched to realize reconfigurable frequency operation [45]. In [46], a $14 \times 17 \mathrm{~mm}^{2}$ ENG unit cell has been used to design a multiband antenna. The addition of a rectangular unit cell in the ground plane makes the monopole antenna useful for triple-band applications. In [47], a split ring resonator-based MTM has been reported. This rectangular-shaped MTM is used in material sensor applications to test concrete materials and utilizes resonant frequency movement as indicated by the parameter that is going be detected. A transmission line incorporated metamaterial sensor has been illustrated in [48]. This sensor is used to detect the authentic and inauthentic diesel samples. Moreover, it can be employed to accurate detection of different liquids. Another omega-shaped resonator along with transmission line has been utilized to realize an MTM operating at $1.9 \mathrm{GHz}$ [49]. This single 
band MTM has used as a hypersensitized liquid sensor and the shifting of the resonant frequency is investigated to determine the concentration of the liquids.

Recent researches on MTMs are intended to improve the effective medium ratio, make it useful for multiband operation and electrical size reduction, etc. In common sense, miniaturization can be done by increasing the value of inductance and capacitance. Increasing the length of the metal stripline and decreasing the capacitive gaps, it is possible to increase the total inductance and capacitance, respectively. This phenomenon paves the way for the size reduction of the meta-atoms. The above-stated paragraph explores many ways of designing conventional and recent ENG MTMs.

Here, the design and performance analysis of a tripleband single negative MTM is reported in this article. A unit cell of D-Z shaped loop is designed to produce multiple operating bands over L-, S-, and X-bands applications. It is well known that metamaterial can be integrated into many microwaves and higher frequency applications for size reduction and performance improvement purpose. $\mathrm{So}$, the proposed multiband unit cell is designed at $\mathrm{L}-, \mathrm{S}-$, and X-bands so that it can be utilized in different microwave applications operating at $\mathrm{L}-, \mathrm{S}-$, and $\mathrm{X}$-bands. The first

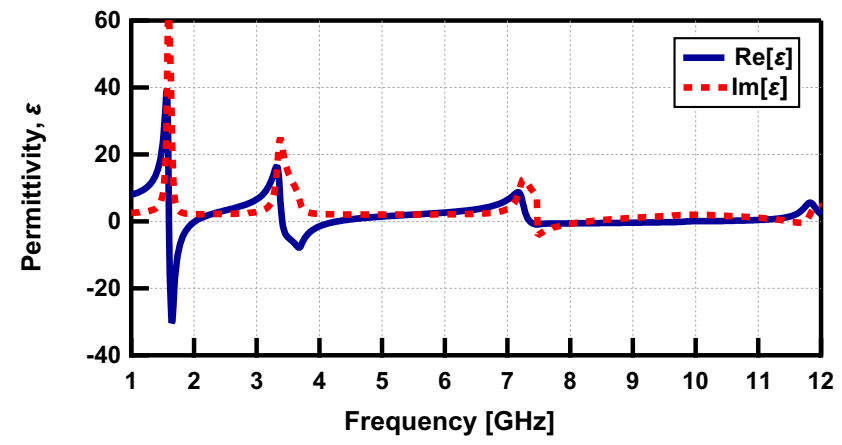

(a) two lower bands are purely electric, and the third band is caused by magnetic resonance. The negative permittivity is extended from $1.54-2 \mathrm{GHz}$ corresponding to an impedance bandwidth of $0.46 \mathrm{GHz}$ for L-band and 3.4-4.2 GHz equivalent to a bandwidth of $0.8 \mathrm{GHz}$ for S-band. The span of the negative permeability region of $X$-band is from 10.32-11.5 GHz equivalent to an impedance bandwidth of $1.18 \mathrm{GHz}$. Moreover, $1 \times 2,2 \times 1,2 \times 2$, and $4 \times 4$ array structures are designed, and investigation of the effective parameters is done. All the structures reveal their tripleband operation, and they are promising for $\mathrm{L}-, \mathrm{S}-$, and $\mathrm{X}$-bands applications. Further, the equivalent circuit for three operating bands is designed and explained numerically. The proposed MTM unit cell exhibits a higher effective medium ratio of 17.7 at $1.54 \mathrm{GHz}$ that proves its compactness for the lower frequency band. So, the projected compact MTM is a prosperous solution for different microwave applications, for example, small antenna design and performance improvement of an antenna for GPS, $5 \mathrm{G}$ and $\mathrm{X}$-band RADAR applications. Moreover, it can be utilized in notch filter design, MTM absorbers, and sensors.

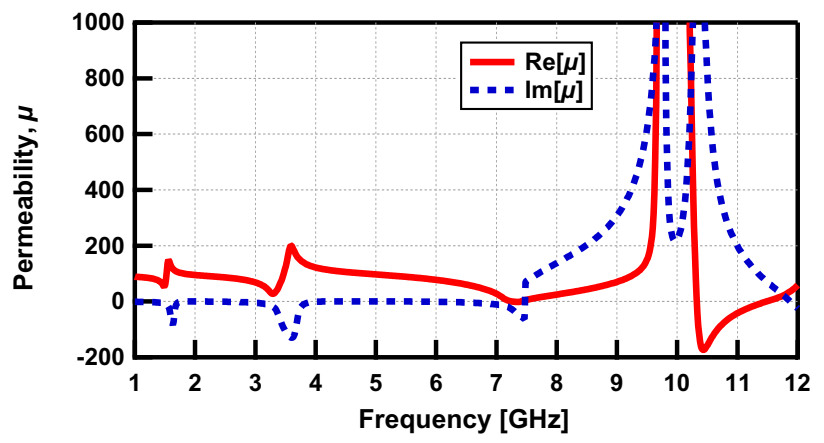

(b)

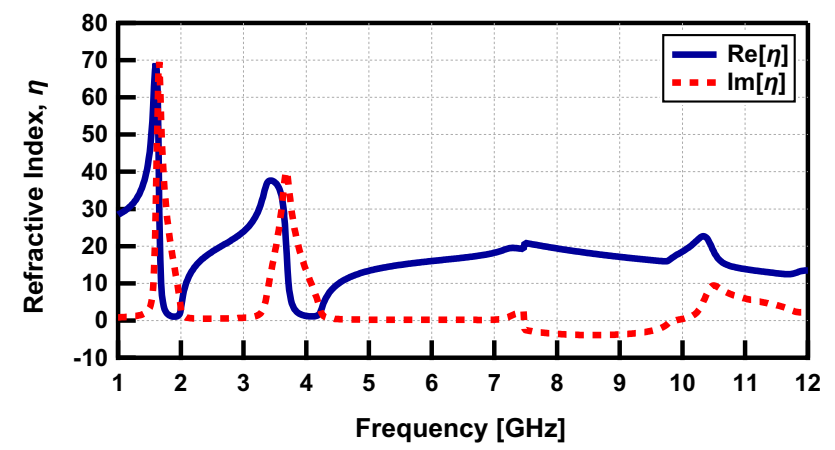

(c)

Fig. 4 Retrieved basic parameters of the proposed triple-band MTM unit cell a Real and imaginary values of permittivity, $\mathbf{b}$ Real and imaginary values of permeability, $\mathbf{c}$ Real and imaginary values of the refractive index 


\section{Design of a triple-band D-Z shaped MTM}

The structure of the prospective D-Z shaped MTM unit cell is depicted in Fig. 1. Here, two open D-shaped split-ring resonators are coupled by a Z-shaped structure. The whole patch is imprinted on the FR4 substrate of permittivity, $\varepsilon_{r}=4.4$, loss tangent $=0.0025$, and a height of $h$. The overall dimension of the unit cell is $11 \times 11 \mathrm{~mm}^{2}$. The outer arm length of the D-shaped resonator is $I$, and inner arm length is $I_{1}$. Two $D$-shaped resonators have two split gaps of $g$ and capacitively coupled by a gap of $t$. The length of the $Z$-shaped structure is $m$. The thickness of the copper strip is $0.035 \mathrm{~mm}$ having a width of $c$. The effective design parameters are enlisted in Table 1. Some elementary points of the simulation setup are as follows: the perfect electric boundary is executed along the $y$-axis, and the $x$-axis is considered as a perfect magnetic boundary. Then the unit cell is placed between two waveguide port, and they are used to EM wave propagation along the positive and negative $z$-axis, as shown in Fig. 1 b. A frequency-domain solver is used for the free space simulation purpose. A tetrahedral mesh setup and port impedance of $50 \Omega$ is used for simulation purposes.

After the simulation, the transmission and reflection coefficient curves are obtained and depicted in Fig. 2. The minimum dip of the transmission coefficient curve indicates the resonant frequency of the proposed MTM. The transmission dips occur at $1.54 \mathrm{GHz}, 3.5 \mathrm{GHz}$, and $10.4 \mathrm{GHz}$. The absorptivity curve of the proposed triple-band MTM is shown in Fig. 3. Absorptivity $(A(f))$ of the MTM unit cell can be evaluated from the $S_{11}$ and $S_{21}$ by using the following equation: Absorption spectra, $A(f)=1-\left|S_{21}(f)\right|^{2}$ - $\left|S_{11}(f)\right|^{2}$. From the absorption spectra, it is shown that it creates three peaks at $1.6 \mathrm{GHz}, 3.6 \mathrm{GHz}$, and $8.7 \mathrm{GHz}$, correspondingly. The maximum values of absorption spectra are $0.2,0.18$ and 0.32 corresponding to $1.6,3.6$, and $8.7 \mathrm{GHz}$, respectively. The absorption spectra $A(f)$ is also known as loss characteristics. The ideal value of loss characteristics is 1 . When the value of loss characteristics is 1 , EM wave neither transmitted nor reflected. Still, it is a blessing for applications like metamaterial absorbers.
Fig. 5 Surface current distribution of the proposed triple-band MTM a $1.54 \mathrm{GHz}$, b $3.5 \mathrm{GHz}, \mathrm{c} 10.4 \mathrm{GHz}$

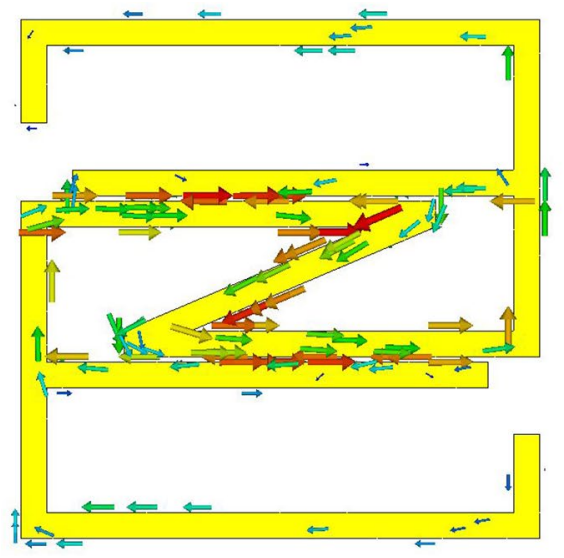

(a)

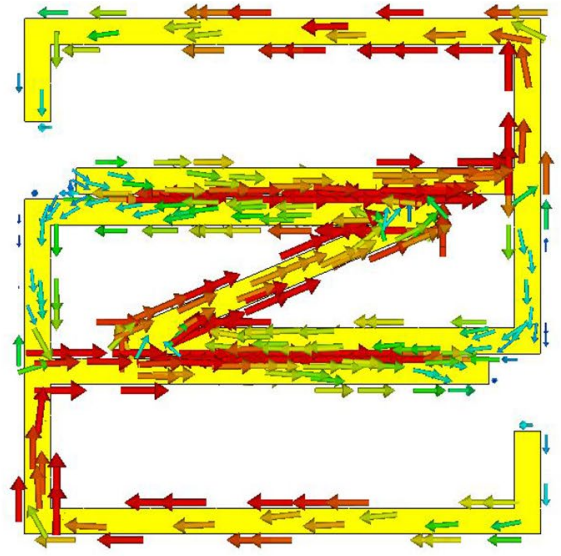

(b)

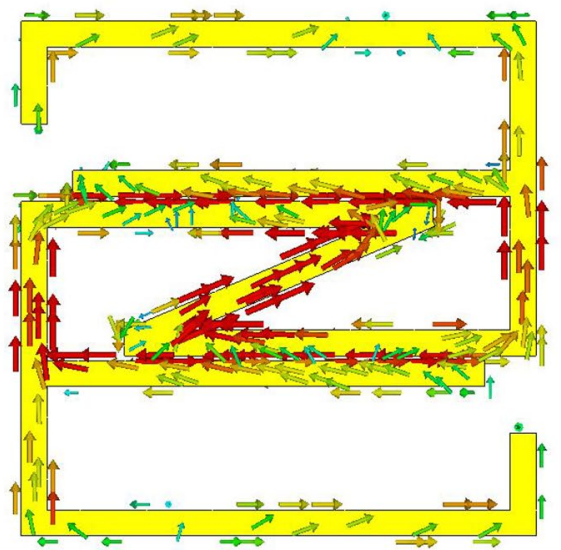

(c) 
Fig. 6 Electric field distribution of the proposed triple-band MTM a $1.54 \mathrm{GHz}$, b $3.5 \mathrm{GHz}$, c $10.4 \mathrm{GHz}$

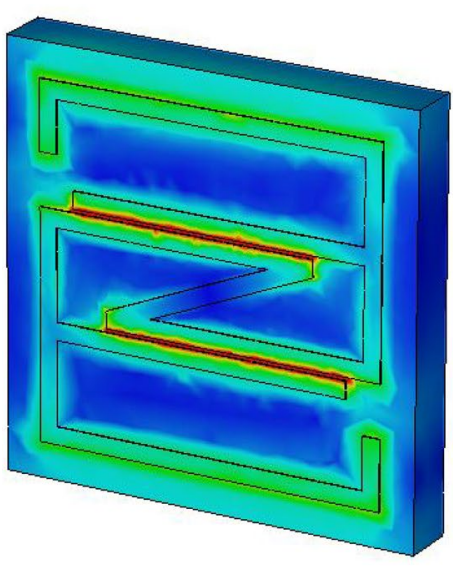

(a)

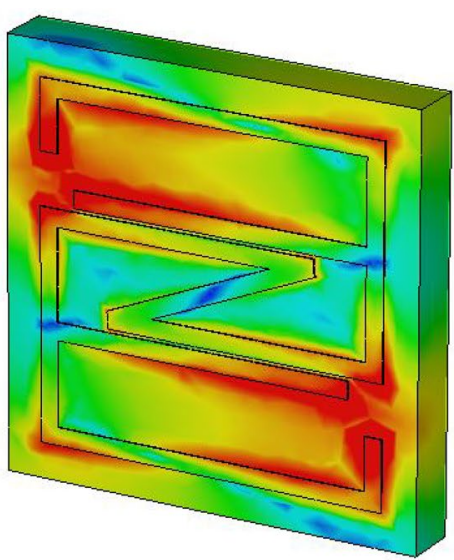

(b)

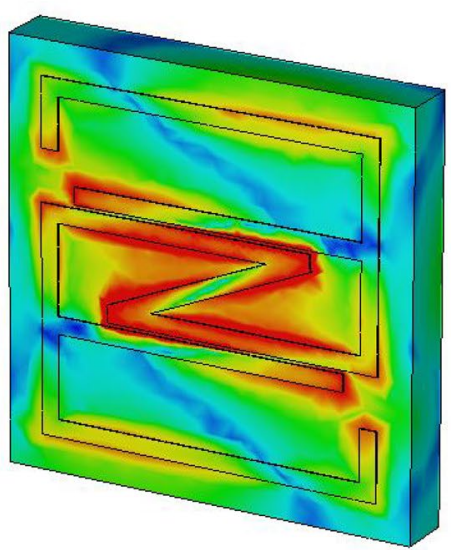

(c)

But the lower value of $A(f)$ is desired in applications such as metamaterial cloaking, metamaterial antenna design, photonic metamaterials, etc. As our proposed metamaterial unit cell is not a metamaterial absorber; the peaks of absorption spectra contain lower values. It can be further lowered by using high-quality substrate and high conductivity metal. Moreover, higher loss characteristics can be obtained by minimizing wave transmission and matching the wave impedance and surface impedance of the metamaterial slab [50].

\section{Parameter abstraction}

To determine the EM behaviour of the MTM unit cell, scattering parameters (reflection and transmission coefficients) are used. To recover the basic parameters of the unit cell such as permittivity, permeability, and refractive index, Nicolson Ross Weir method (NRW) is used and required that the MTM unit cell should be smaller in size than the guided wavelength $[51,52]$. The reflection and transmission coefficients can be expressed in the term as follows:
$S_{11}=\frac{\left(1-z^{2}\right) \Gamma}{1-\Gamma^{2} z^{2}}$

$S_{21}=\frac{\left(1-\Gamma^{2}\right) z}{1-\Gamma^{2} z^{2}}$

where $\Gamma$ is termed as reflection coefficient between the substrate of thickness $h$ and free space. Further, $\Gamma$ can be expressed in the term of relative impedance $Z_{0}$ (relative impedance) and it is the ratio between the square root of relative permeability and permittivity. The remaining parameter $z$ is the transmission coefficient which is related to the substrate of thickness $h$, the velocity of light $c$, and angular frequency of $\omega(\omega=2 \pi f)$.

$$
\begin{aligned}
& \Gamma=\frac{\left(Z_{0}-1\right)}{\left(Z_{0}+1\right)} \\
& Z_{0}=\frac{\sqrt{\mu_{r}}}{\sqrt{\varepsilon_{r}}}
\end{aligned}
$$


Fig. 7 Equivalent circuit of the proposed triple-band MTM a

$1.54 \mathrm{GHz}$, b $3.5 \mathrm{GHz}, \mathbf{c} 10.4 \mathrm{GHz}$

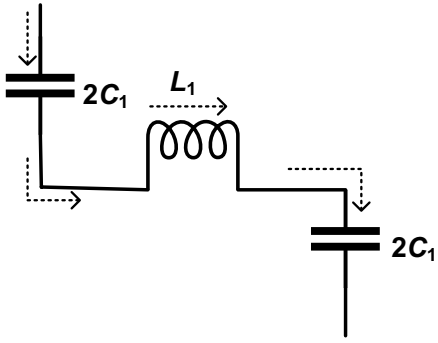

(a)

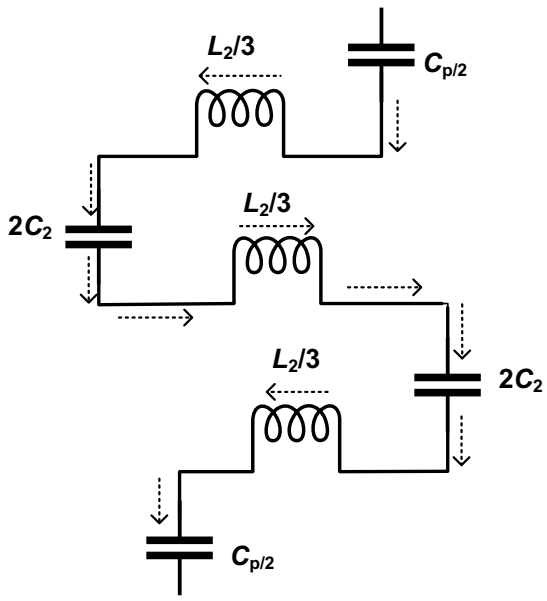

(b)

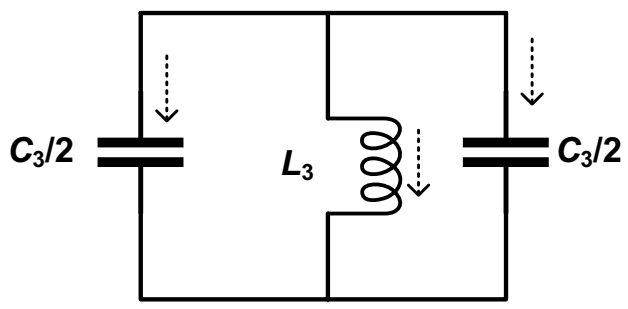

(c)

If $X_{1}=S_{21}+S_{11}$ and $X_{2}=S_{21}-S_{11}$ then according to NRW method effective permittivity $\left(\varepsilon_{\text {eff }}\right)$, permeability $\left(\mu_{\text {eff }}\right)$ and refractive index $\left(\eta_{\text {eff }}\right)$ can be expressed as follows:

$\varepsilon_{e f f}=\frac{2 c}{j \omega d} \times \frac{\left(1-X_{1}\right)}{\left(1+X_{1}\right)}$

$\mu_{\text {eff }}=\frac{2 c}{j \omega d} \times \frac{\left(1-X_{2}\right)}{\left(1+X_{2}\right)}$

$\eta_{\text {eff }}=\sqrt{\mu_{\text {eff }} \varepsilon_{\text {eff }}}$

The extracted real and imaginary values of permittivity, permeability and refractive index at $1.54 \mathrm{GHz}, 3.5 \mathrm{GHz}$, and $10.4 \mathrm{GHz}$ are illustrated in Fig. 4. The proposed tripleband MTM resonates in three frequency bands having two electric and one magnetic resonance. At each resonant band, the imaginary components of permittivity and permeability are positive. From positive and negative-going real values of permittivity, it is clear that electric resonance occurs at $1.54 \mathrm{GHz}$ and $3.5 \mathrm{GHz}$. The negative permittivity region ranges from $1.54-2 \mathrm{GHz}$ and $3.4-4.2 \mathrm{GHz}$ for the first and second band, respectively. The existence of negative permeability can easily be seen in Fig. 4b. The negative permeability region ranges from $10.32-11.5 \mathrm{GHz}$. As only one effective parameter $(\varepsilon, \mu)$ is negative at a time, the refractive index is positive, as shown in Fig. 4c.

\section{Surface current and electric field distribution of proposed MTM unit cell}

To reach deeper inside the operation of triple-band MTM, surface current distribution, and electric field distribution at three distinct operating bands are analyzed and figured in Figs. 5, and 6. At $1.54 \mathrm{GHz}$, the surface current flows in the upper, lower and inclined part of the Z-shaped structure. In the upper and lower part of the Z-shaped structure, the clockwise and anticlockwise movement of surface current annulled the magnetic dipole activity as pictured in Fig. 5a. The electric field distribution at $1.5 \mathrm{GHz}$, as presented in Fig. 6a indicates that the upper and lower part of the Z-shaped structure capacitively coupled with a D-shaped structure. When the capacitor discharged, current flows through the inclined metal strip of the Z-shaped structure and can be modelled as an inductor. For the second resonant frequency at $3.5 \mathrm{GHz}$, 


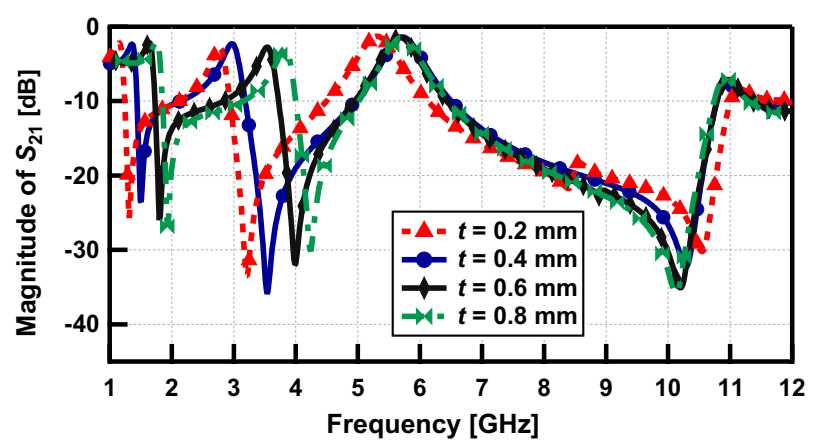

(a)

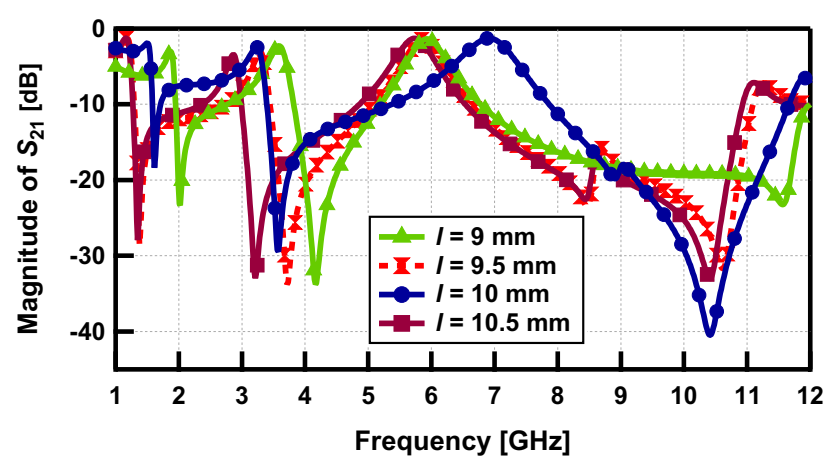

(c)

Fig. 8 Parametric analysis by altering $\mathbf{a} t, \mathbf{b} g, \mathbf{c} l$, and $\mathbf{d} c$

the concentration of the electric field is strong near the opening of the D-shaped loop and is depicted in Fig. 6 b. The magnitude of the surface current is remarkable in the upper and lower part of the D-shaped loop and also in the inclined part of the Z-shaped metal strip at $3.5 \mathrm{GHz}$ frequency. This phenomenon is portrayed in Fig. $5 \mathrm{~b}$. For the L-band and S-band resonant frequencies, the applied electric field is in phase with the current induced in the MTM. So, only the permittivity becomes negative.

Figure 4a shows that the negative permittivity region extended from 1.54-2 $\mathrm{GHz}$ equivalent to a bandwidth of $0.46 \mathrm{GHz}$ and $3.4-4.2 \mathrm{GHz}$ corresponding to a bandwidth of $0.8 \mathrm{GHz}$. But in the case of third resonant frequency, a phase change occurs. To cover up the fluctuating magnetic field, a change in current path occurs, and the current lags the applied electric field. Hence, negative permeability creates at $10.4 \mathrm{GHz}$. The region of negative permeability ranges from $10.32-11.5 \mathrm{GHz}$. The surface current distribution and electric field distribution at $10.4 \mathrm{GHz}$ are shown in Figs. 5c and 6c.

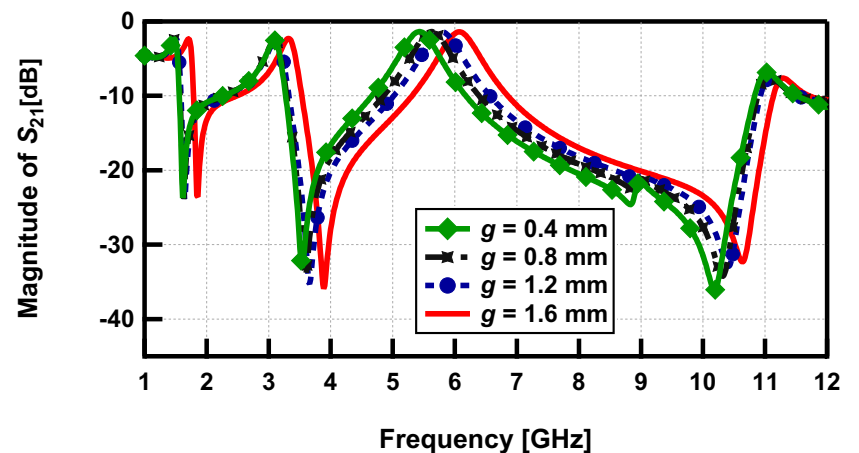

(b)

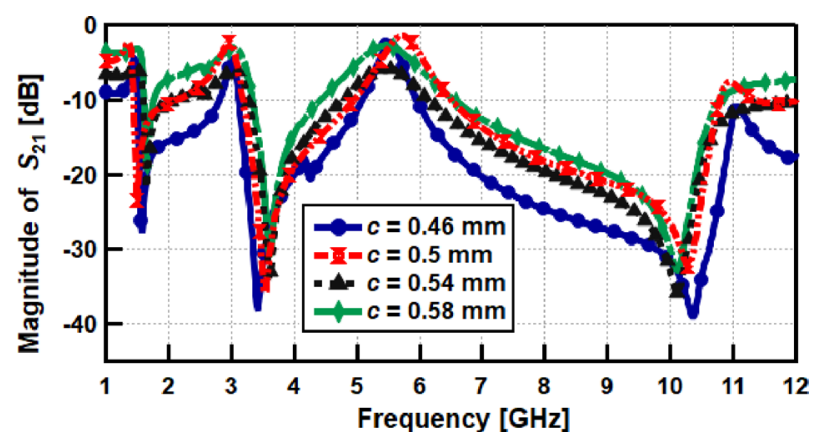

(d)

\section{Equivalent circuit design}

The investigation of an equivalent circuit at each band of the proposed triple-band MTM makes its operation mechanism easier to understand. The equivalent circuit of an MTM can be estimated as LC resonant circuit, especially in quasistatic boundary (when unit cells are much smaller). The common formula of the resonant frequency is $f=1 / 2 \pi \sqrt{L C}$, where $L$ is inductance and $C$ is capacitance.

In MTM design, the current produced in metallic strip offers inductance, and the gap between two copper strip causes capacitance. The resonant frequency can be extended and lower by changing the value of $L$ and $C$. The proposed unit cell is excited by the perpendicularly incident polarized wave and causes an introduction of voltage in the metal gaps or parallel metal strip. This phenomenon can be approximated with the formation of parallel plate capacitance. Moreover, inductance can be modelled by the discharging path of the capacitor. The equivalent circuits are shown in Fig. 7. From the previous analysis of the surface current and electric field distribution, the responsible parts of the unit cell for capacitance and inductance generation are highlighted. If the width of the metal strip is $c$, the spacing between the capacitive gaps are $g$ and $t$, and the thickness of the 
substrate is $h$ then the inductance and capacitance can be calculated by using formulas illustrated in the next paragraph $[53,54]$.

The capacitance form between two gaps can be expressed as

Capacitance, $C=\varepsilon_{0} \varepsilon_{\text {eff }} \frac{k\left(k_{0}^{\prime}\right)}{k\left(k_{0}\right)}$

where $\varepsilon_{\text {eff }}$ is the effective dielectric constant and related to fill factor $q$. Therefore,

$$
\begin{aligned}
\varepsilon_{\text {eff }} & =1+(\varepsilon-1) q \\
q & =\left(\frac{1}{2}\right)\left(\frac{K\left(k^{\prime}\right)}{K(k)}\right)\left(\frac{K\left(k_{0}\right)}{K\left(k_{0}^{\prime}\right)}\right), \quad k_{0}=\frac{\sinh \left(\frac{\pi s}{2 h}\right)}{\sinh \left(\frac{\pi b}{2 h}\right)} \\
k_{0}^{\prime} & =\sqrt{\left(1-k_{0}^{2}\right)}, k=\frac{s}{b}, k^{\prime}=\sqrt{1-k^{2}}, s=\frac{g}{2}, b=\frac{g}{2}+c
\end{aligned}
$$

where $K$ is the elliptical function of the first kind. Now, expression of inductance can be expressed by:

Fig. 9 Geometrical configurations of arrays a $1 \times 2, \mathbf{b} 2 \times 1, \mathbf{c}$ $2 \times 2$, and $\mathbf{d} 4 \times 4$ array

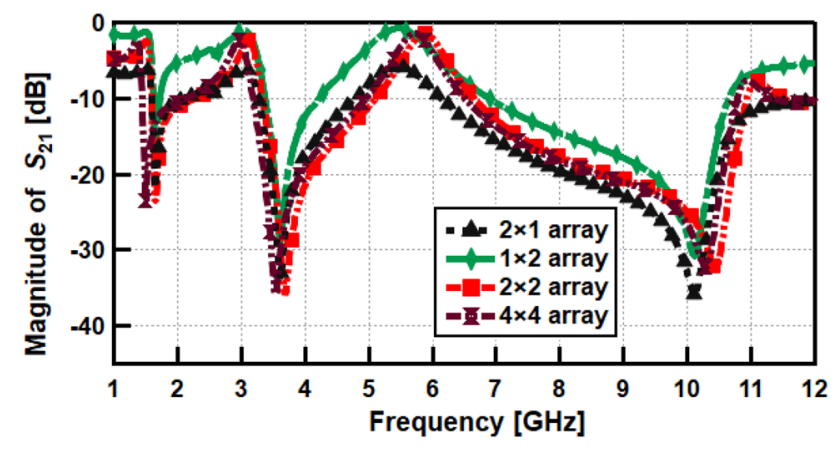

Fig. $10 S_{21}$ curves of the array configurations

Inductance, $L=\left(\frac{\sqrt{(2 c+h)^{2}+l^{2}}}{c}+\frac{2 c}{2 w+h}\right) \mu_{0} h$

As stated before, the lower resonant frequency at $1.54 \mathrm{GHz}$ is due to capacitive coupling between $\mathrm{D}$ - and $Z$-shaped structure and also for the discharge path of

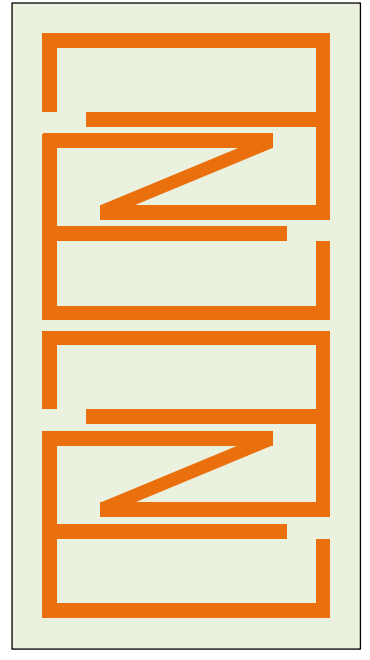

(a)

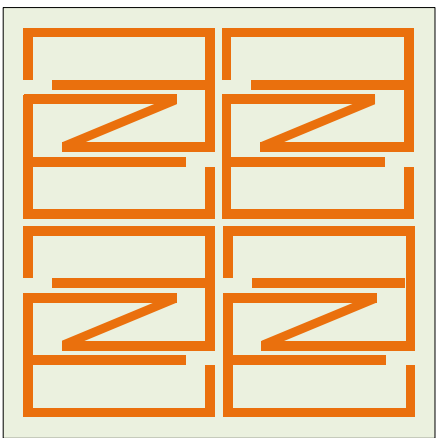

(c)

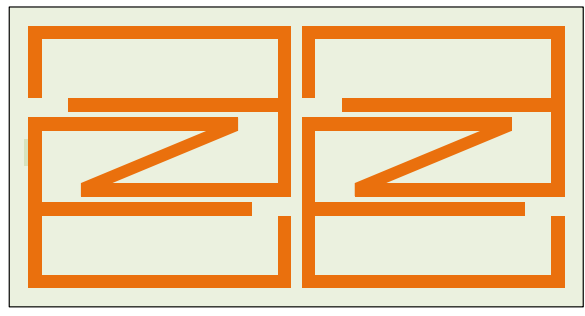

(b)

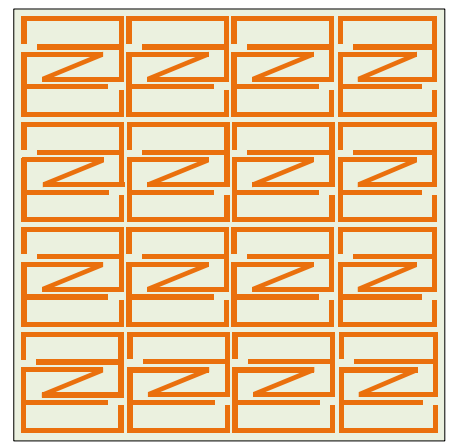

(d) 


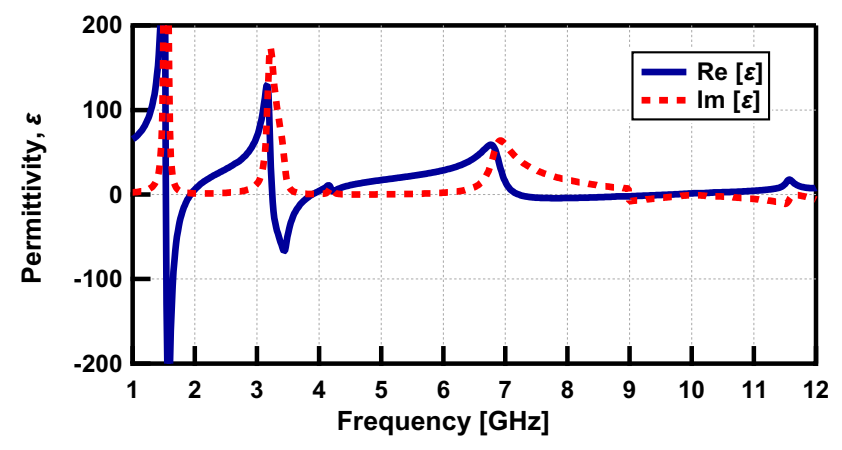

(a)

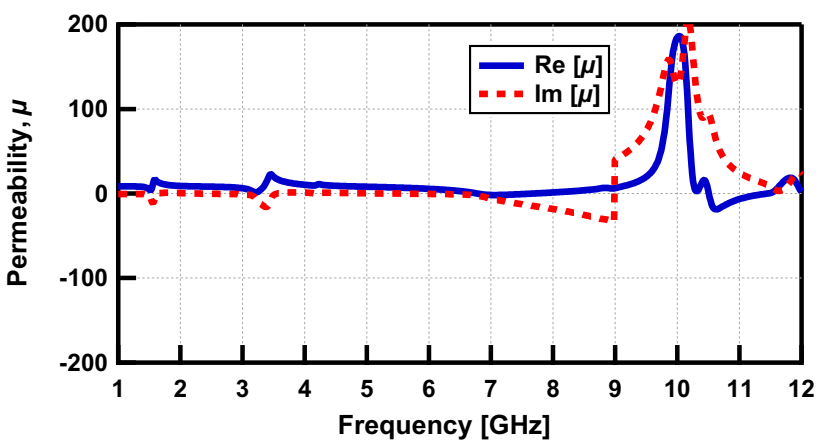

(b)

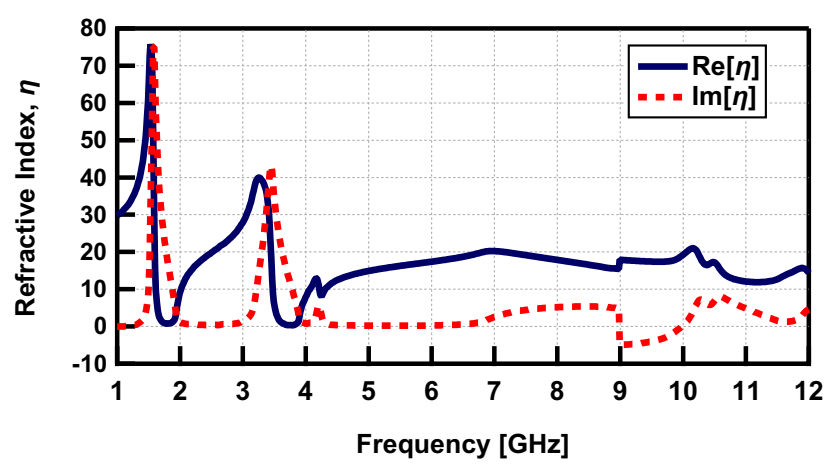

(c)

Fig. $111 \times 2$ array configuration a effective permittivity, $\mathbf{b}$ effective permeability, and $\mathbf{c}$ refractive index

the capacitor (the inclined metal strip). The calculated value of the capacitance, $C_{1}=4.63 \mathrm{pF}$ and inductance, $L_{1}=2.566 \mathrm{nH}$. So, the calculated first resonant frequency $f_{1}=1.46 \mathrm{GHz}$, which is very close to the simulated resonance frequency of $1.54 \mathrm{GHz}$. The creation of the second resonant frequency at $3.5 \mathrm{GHz}$ is related to the capacitance formation in the right and left part of the open $D$ loop and the current flowing through the upper and lower part of the proposed structure. After calculation, the approximated value of capacitance, $C_{2}=12.2 \mathrm{pF}$ and inductance, $L_{2}=0.24 \mathrm{nH}$ hence the second calculated resonant frequency is $f_{2}=3.39 \mathrm{GHz}$. In this case, the effects of the intercell capacitance $\left(C_{p}\right)$ are neglected. The calculated resonant frequency for the third frequency $f_{3}=9.989 \mathrm{GHz}$ where capacitance $C_{3}=9.15 \mathrm{pF}$ and inductance $L_{3}=0.028$ $\mathrm{nH}$.

\section{Effect of varying structure parameters on the resonant frequency}

To find the significance of various design parameter, a parametric analysis is performed. Several simulations are done by varying capacitive gaps $g$ and $t$, length $l$, and width of the metal strip $c$. The parametric analysis will help to figure out the resonance mechanism and also the design procedure.

Figure 8 represents the transmission spectra after the variation of the design parameters. The capacitive gap $t$ is altered from 0.2 to $0.8 \mathrm{~mm}$, and respective transmission spectra are illustrated in Fig. 8a. The optimized value of gap $t$ is chosen to $0.4 \mathrm{~mm}$, where three resonances occur at $1.54 \mathrm{GHz}, 3.5 \mathrm{GHz}$, and $10.4 \mathrm{GHz}$. When the value of $t$ is reduced to $0.2 \mathrm{~mm}$, the first resonant frequency reduced to $1.33 \mathrm{GHz}$. Because the capacitive gap decreased for the first resonant band. The second operating frequency band slightly decreased at $t=0.4 \mathrm{~mm}$ and the third band increased. This happens probably due to the stronger effect of $C_{2}$ and the weaker effect of $C_{3}$. Similarly, due to the extending the value of $t$ beyond $0.4 \mathrm{~mm}$ causes first resonant increases.

To point out the effect of capacitive gap $g$ on the resonant frequency of the proposed structure, a parametric analysis is done. Here, the gap $g$ is varied in a step of $0.4 \mathrm{~mm}$ and from 0.4 to $1.6 \mathrm{~mm}$ as depicted in Fig. $8 \mathrm{~b}$. From the figure, it is clear that an increase in $g$ causes an increase in second resonant frequency and vice versa. The first resonant band is also affected by the variations of gap $g$ because electric field concentration altered significantly within gap $t$. The change of third 


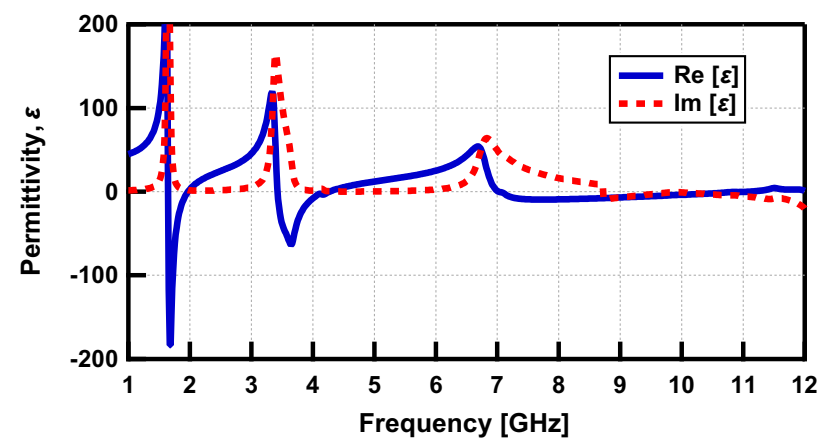

(a)

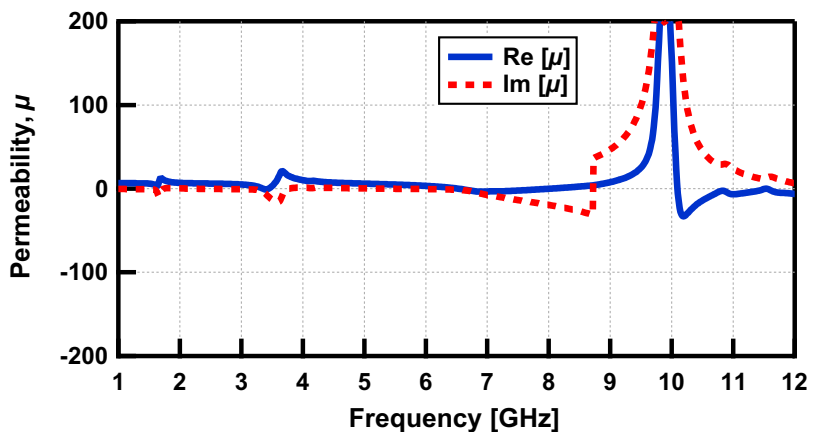

(b)

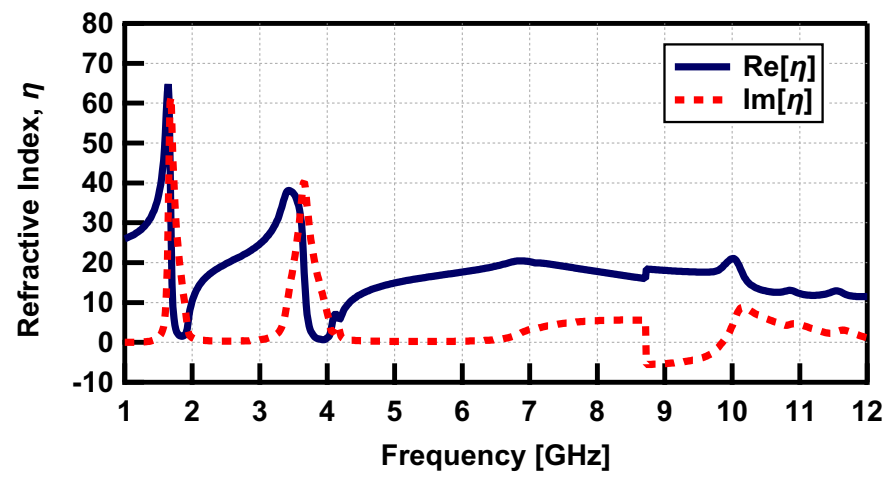

(c)

Fig. $122 \times 1$ array configuration a effective permittivity, $\mathbf{b}$ effective permeability, and $\mathbf{c}$ refractive index

resonance frequency is negligible up to a gap value of $0.4 \mathrm{~mm}$ to $1.2 \mathrm{~mm}$. At $g=1.6 \mathrm{~mm}$, the increase in the third resonance frequency band is due to the decrease in the inductive path length. So, the optimum value of $g$ is selected as $1.2 \mathrm{~mm}$.

A large value of inductance is possible by increasing the length of the metal strip. As a result, the shifting of resonance frequencies will be downwards. This phenomenon is observed in Fig. 8c, where lengthening the metal strip causes a decrease in resonance and vice versa. The optimized value of length / is $10 \mathrm{~mm}$.

The resonant frequency doesn't change significantly by the variation of the width of metal strip $c$. From Fig. 8d it can be observed easily. The variation of the first and second resonant band is poor by the variation of $c$. But the third band altered in a small range. This is due to the change of the mutual inductance of the proposed structure. In this worked design, the value of the optimized metal strip width is $0.5 \mathrm{~mm}$ because it is well known that the conductor losses are larger in a thin strip.

\section{Design and analysis of different array structure}

To improve the performance of an antenna using MTM, it is sometimes necessary to use them in an array style. This section explores the behaviour of the proposed tripleband MTM when used in an array configuration. If the the array structure is able to create three resonating frequency bands with a negative value of effective parameters then it can be concluded that the proposed MTM structure can be applied for design and performance improvement of the antenna. The geometrical configurations of $1 \times 2$, $2 \times 1,2 \times 2$, and $4 \times 4$ arrays are shown in Fig. 9 . The distance between the two cells is $0.5 \mathrm{~mm}$. The overall dimensions are $11 \times 22 \mathrm{~mm}^{2}, 22 \times 11 \mathrm{~mm}^{2}, 22 \times 22 \mathrm{~mm}^{2}$, and $44 \times 44 \mathrm{~mm}^{2}$ corresponding to $1 \times 2,2 \times 1,2 \times 2$, and $4 \times 4$ array structures of the unit cell. The $S_{21}$ curves of the array configurations are listed in Fig. 10. Figure 10 illustrates that array configurations are also able to resonate in three frequency bands. The $1 \times 2$ array shows triple-band operation at $1.6 \mathrm{GHz}$, $3.55 \mathrm{GHz}$, and $10.2 \mathrm{GHz}$, but the magnitude of $S_{21}$ is higher 


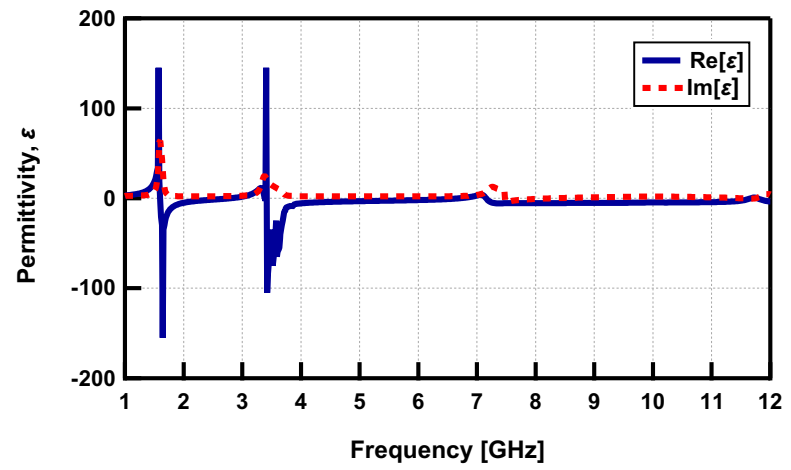

(a)

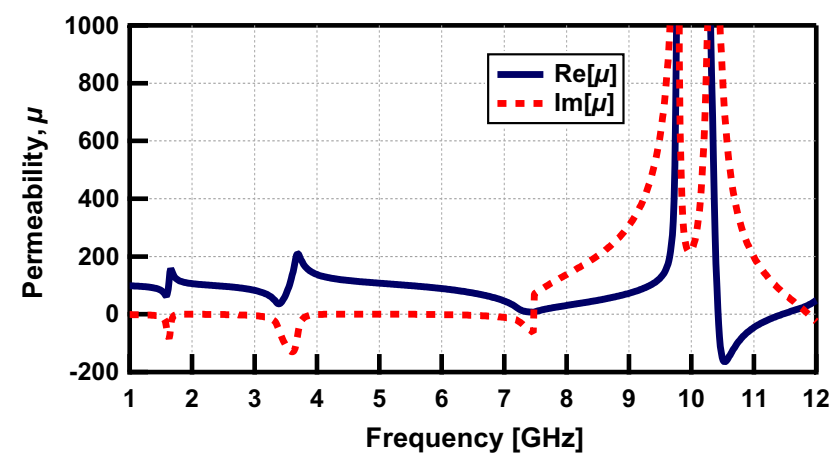

(b)

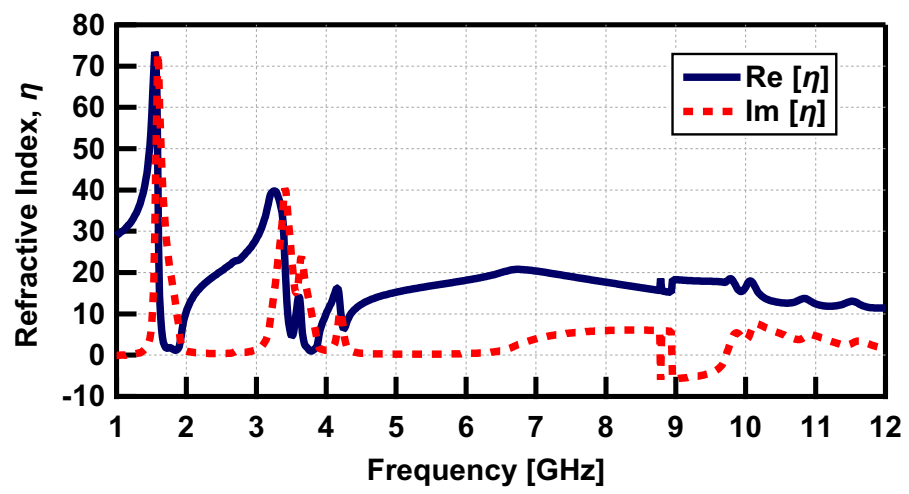

(c)

Fig. $132 \times 2$ array configuration a effective permittivity, $\mathbf{b}$ effective permeability, and $\mathbf{c}$ refractive index

for the first and second frequency band. As illustrated in Fig. 11, the negative region is found from $1.5-1.9 \mathrm{GHz}$ to 3.2-4.8 GHz for permittivity and for permeability the range is from $10.6-11.4 \mathrm{GHz}$. The $2 \times 1$ array exhibits three operating frequency bands at $1.6 \mathrm{GHz}, 3.55 \mathrm{GHz}$, and $10.1 \mathrm{GHz}$, respectively. The negative permittivity region ranges from 1.6-2 GHz and 3.4-4.3 GHz, where the negative permeability region extended from $10.1-11.5 \mathrm{GHz}$ and depicted in Fig. 12. The triple-band performance is also observed in the $2 \times 2$ array structure, and the transmission minimums are at $1.67 \mathrm{GHz}, 3.67 \mathrm{GHz}$, and $10.45 \mathrm{GHz}$, correspondingly. From Fig. 13, the negative permittivity and permeability characteristics are observed from $1.6-2.6 \mathrm{GHz}, 3.4-5 \mathrm{GHz}$, and $10.4-11.2 \mathrm{GHz}$, respectively. Finally, the largest $4 \times 4$ array is also able to generate three transmission minima at $1.5 \mathrm{GHz}, 3.5 \mathrm{GHz}$, and $10.3 \mathrm{GHz}$, respectively. The effective values of permittivity and permeability are represented in Fig. 14. The SNG characteristics are remarkable from 1.5-2 GHz, 3.2-4.7 GHz, and $10.1-11.2 \mathrm{GHz}$ frequency ranges. The real and imaginary refractive index curves of $1 \times 2,2 \times 1,2 \times 2$, and $4 \times 4$ array structures are shown in Figs. 11c, 12c, 13c, and 14c, respectively. None of them can show NRI properties as simultaneous values of $\varepsilon$ and $\mu$ are not negative. The refractive index of the first two bands is close to zero, and the third band has a higher positive value of the refractive index. A summary of negative effective parameters is listed in Table 2, showing the negative regions, application bands, operating frequencies, and relative bandwidths corresponding to the structure types.

\section{Comparative study}

A comparative study is performed to compare the proposed work with recently announced MTM structures and appeared in Table 3. The comparison is based on the overall size of the unit cell, the effective medium ratio, and based on operating bands. In contrast with the literature [44], the proposed design is compact, having a higher value of the effective medium ratio, at $1.54 \mathrm{GHz}$. Compared to $[32,38,43,45]$ the size of the worked design is compact as it offers three operating bands. The design is applicable for lower frequency regions with miniature size compared to the literature [46]. Moreover, none of the listed work can operate in a lower frequency band $(1.54 \mathrm{GHz})$ with a higher value of the effective medium ratio. 


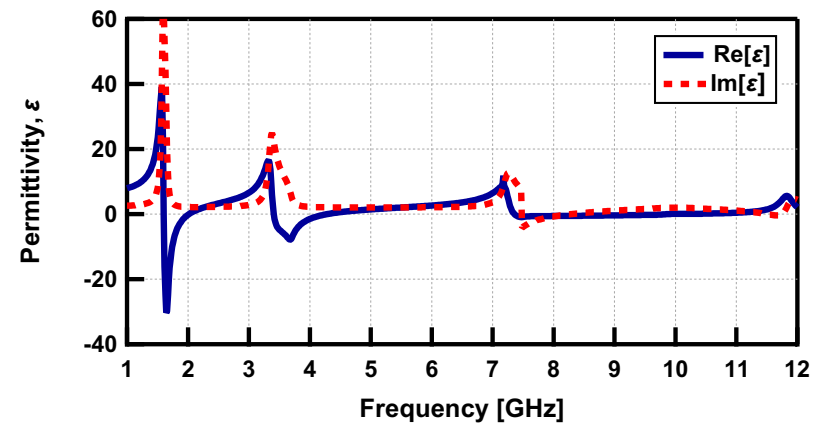

(a)

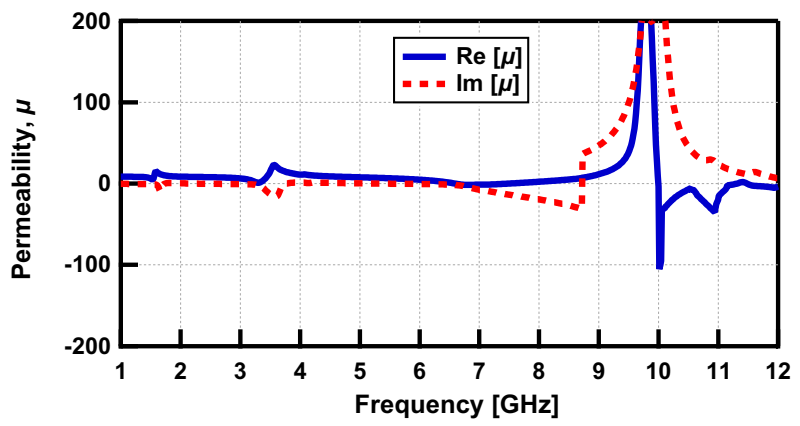

(b)

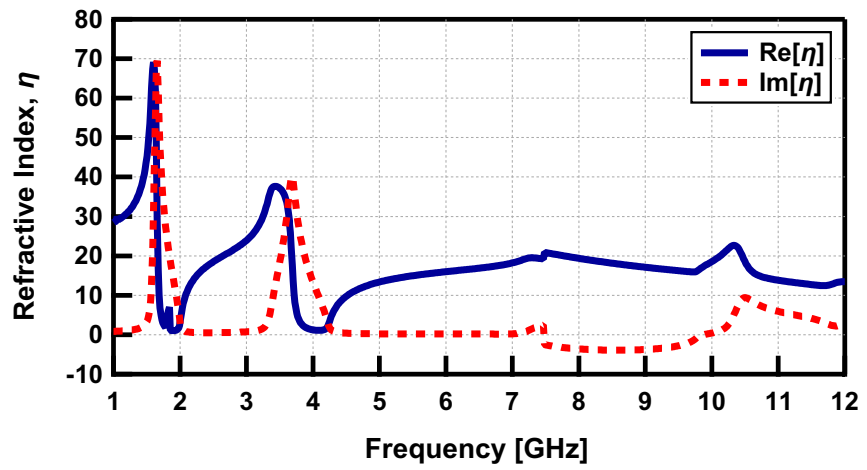

(c)

Fig. $144 \times 4$ array configuration a effective permittivity, $\mathbf{b}$ effective permeability, and $\mathbf{c}$ refractive index

Table 2 A summary of the frequency range, bandwidth, and application bands covered by proposed configurations established on negative permittivity and permeability

\begin{tabular}{|c|c|c|c|c|}
\hline Effective parameters & Configurations & Frequency range $(\mathrm{GHz})$ & Bandwidth (GHz) & $\begin{array}{l}\text { Application } \\
\text { bands }\end{array}$ \\
\hline \multirow[t]{10}{*}{ Permittivity } & Unit cell & $1.54-2$ & 0.46 & L, S \\
\hline & & $3.4-4.2$ & 0.8 & \\
\hline & $1 \times 2$ array & $1.5-1.9$ & 0.4 & $L, S$ \\
\hline & & $3.2-4.8$ & 1.6 & \\
\hline & $2 \times 1$ array & $1.6-2$ & 0.4 & $L, S$ \\
\hline & & $3.4-4.3$ & 0.9 & \\
\hline & $2 \times 2$ array & $1.6-2.6$ & 1 & $L, S, C$ \\
\hline & & $3.4-5$ & 1.6 & \\
\hline & $4 \times 4$ array & $1.5-2$ & 0.5 & $L, S$ \\
\hline & & $3.2-4.7$ & 1.5 & \\
\hline \multirow[t]{5}{*}{ Permeability } & Unit cell & $10.32-11.5$ & 1.18 & $\mathrm{X}$ \\
\hline & $1 \times 2$ array & $10.6-11.4$ & 0.8 & $x$ \\
\hline & $2 \times 1$ array & $10.1-11.5$ & 1.4 & $x$ \\
\hline & $2 \times 2$ array & $10.4-11.2$ & 0.8 & $X$ \\
\hline & $4 \times 4$ array & $10.1-11.2$ & 1.1 & $x$ \\
\hline
\end{tabular}


Table 3 A comparison among the proposed MTM unit cell and other works of literature

\begin{tabular}{lllllll}
\hline References & Year & Size $\left(\mathrm{mm}^{2}\right)$ & Frequency $(\mathrm{GHz})$ & Frequency band & Effective medium ratio & Configuration \\
\hline$[32]$ & 2010 & $14 \times 14$ & 2.11 & $\mathrm{~S}$ & 10.2 & Integrated Capacitor loaded ELC resonator \\
{$[37]$} & 2015 & $7.5 \times 7.5$ & $5.41,6.70$ & $\mathrm{C}$ & $7.39,5.96$ & Square and meander loaded delta \\
{$[38]$} & 2015 & $10 \times 10$ & $2.4,3.5,4$ & $\mathrm{~S}$ & $12.5,8.75, \& 7.5$ & Greek-key-pattern \\
{$[43]$} & 2015 & $10 \times 10$ & 8.29 & $\mathrm{X}$ & 3.58 & Pi- shaped structure \\
{$[44]$} & 2018 & $22 \times 22$ & $1.05,1.15,1.5$ & $\mathrm{~L}$ & $12.9,11.8, \& 9.01$ & Fractal \\
{$[45]$} & 2017 & $9 \times 9$ & 2.24 & $\mathrm{~S}$ & 14.8 & Circular ELC resonator \\
{$[46]$} & 2013 & $14 \times 17$ & $2.5,5.8$ & $\mathrm{~S}, \mathrm{C}$ & $8.5,3.69$ & Rectangular \\
Proposed work & 2019 & $11 \times 11$ & $1.54,3.5,10.4$ & L, S,X & $17.7,7.8, \& 2.63$ & D-Z shaped \\
\hline
\end{tabular}

\section{Conclusion}

A framework of a triple-band single negative MTM has been represented in this article. To design the multiband MTM unit cell, a D-Z shaped structure is used. The proposed SNG MTM is applicable for L-, S-, and X-bands applications. The L-band and S-band resonance are caused by electric resonance, whereas the X-band resonance is generated by magnetic resonance of the proposed structure. Moreover, for a superior understanding of the proposed structure, equivalent circuit modelling is performed at three operating frequencies based on the electric field and surface current distribution. Additionally, the parametric analysis of the unit cell assists in designing tunable MTM. To investigate the behaviour of the triple-band MTM when they have to be used as a bulk, array analysis is also performed. The design and performance analysis of $1 \times 2,2 \times 1,2 \times 2$, and $4 \times 4$ array structures of the proposed unit cells is also performed. However, the array structures showed good assurance of negative parameters. Compared to conventional SNG thin wire and complementary resonators, the proposed triple-band MTM showed improved performance. The main advantages of the proposed SNG MTM design lie in its miniature size, triple-band activity, and having improved value of the effective medium ratio. This proposed design can be beneficial for microwave and higher frequency applications such as in antennas, filters, MTM sensors, and MTM absorbers.

Acknowledgements Authors are grateful to Dr. Ahmed Toaha Mobashsher, School of Information Technology and Electrical Engineering, The University of Queensland, Australia, for his valuable suggestion regarding the simulation of the design.

\section{Compliance with ethical standards}

Conflict of interest On behalf of all authors, the corresponding author states that there is no conflict of interest.

\section{References}

1. Bowen PT, Baron A, Smith DR (2016) Theory of patch-antenna metamaterial perfect absorbers. Phys Rev A 93:063849-(1-8). https://doi.org/10.1103/PhysRevA.93.063849

2. II Kwak S, Sim D-U, Kwon JH, Yoon YJ (2016) Design of PIFA with metamaterials for body-SAR reduction in wearable applications. IEEE Trans Electromagn Compat 59:297-300. https://doi. org/10.1109/TEMC.2016.2593493

3. Kundtz N, Gaultney D, Smith DR (2010) Scattering crosssection of a transformation optics-based metamaterial cloak. New J Phys 12:043039-(1-9). https://doi.org/10.1088/13672630/12/4/043039

4. Fang C, Gao J, Liu H (2014) A novel metamaterial filter with stable passband performance based on frequency selective surface. AIP Adv 4:077114-(1-8). https://doi.org/10.1063/1.48901 08

5. Alam J, Faruque MRI, Islam MT (2018) Labyrinth double split open loop resonator based bandpass filter design for $\mathrm{S}, \mathrm{C}$ and X-band application. J Phys D Appl Phys 51:265102 (1-11). https://doi.org/10.1088/1361-6463/aac569

6. Lin C-C, Ziolkowski RW, Nielsen JA, Tanielian MH, Holloway CL (2010) An efficient, low profile, electrically small, threedimensional, very high frequency magnetic EZ antenna. Appl Phys Lett 96:104102 (1-3). https://doi.org/10.1063/1.3357430

7. Islam M, Islam MT, Samsuzzaman M, Faruque MRI (2015) Compact metamaterial antenna for UWB applications. Electron Lett 51:1222-1224. https://doi.org/10.1049/el.2015.2131

8. Veselago VG (1968) the electrodynamics of substances with simultaneously negative values of $\epsilon$ and $\mu$. Soviet Phys Uspekhi 10:509-514. https://doi.org/10.1070/PU1968v010n04ABEH003 699

9. Smith DR, Padilla WJ, Vier D, Nemat-Nasser SC, Schultz S (2000) Composite medium with simultaneously negative permeability and permittivity. Phys Rev Lett 84:4184-4187. https://doi. org/10.1103/PhysRevLett.84.4184

10. Pendry JB, Holden A, Stewart W, Youngs I (1996) Extremely low frequency plasmons in metallic mesostructures. Phys Rev Lett 76:4773-4476. https://doi.org/10.1103/PhysRevLett.76.4773

11. Pendry JB, Holden AJ, Robbins DJ, Stewart W (1999) Magnetism from conductors and enhanced nonlinear phenomena. IEEE Trans Microw Theory Tech 47:2075-2084. https://doi. org/10.1109/22.798002

12. Caloz C, Sanada A, Itoh T (2004) A novel composite right-/lefthanded coupled-line directional coupler with arbitrary coupling level and broad bandwidth. IEEE Trans Microw Theory Tech 52:980-992. https://doi.org/10.1109/TMTT.2004.823579 
13. Shelby RA, Smith DR, Schultz S (2001) Experimental verification of a negative index of refraction. Science 292:77-79. https://doi. org/10.1126/science.1058847

14. Li D, Szabó Z, Qing X, Li E-P, Chen ZN (2012) A high gain antenna with an optimized metamaterial inspired superstrate. IEEE Trans Antennas Propag 60:6018-6023. https://doi.org/10.1109/ TAP.2012.2213231

15. Mendhe SE, Kosta Y (2014) Gain enhancement and broadband using helical resonating metamaterial superstrate in stacked microstrip patch antenna. Microw Opt Technol Lett 56:19781982. https://doi.org/10.1002/mop.28544

16. Hou QW, Su YY, Zhao XP (2014) A high gain patch antenna based PN zero permeability metamaterial. Microw Opt Technol Lett 56:1065-1069. https://doi.org/10.1002/mop.28261

17. Kim J, Yang D (2013) AN ENG ZOR unit cell antenna using spiral inductors with enhanced bandwidth. Microw Opt Technol Lett 55(3):567-571. https://doi.org/10.1002/mop.27382

18. Daniel RS, Pandeeswari R, Raghavan S (2018) A miniaturized printed monopole antenna loaded with hexagonal complementary split ring resonators for multiband operations. Int J RF Microw Comput-Aided Eng 28:1-8. https://doi.org/10.1002/ mmce.21401

19. Bala BD, Rahim MKA, Murad NA (2014) Dual mode metamaterial antenna for wideband applications. Microw Opt Technol Lett 56:1846-1850. https://doi.org/10.1002/mop.28471

20. Zhu J, Antoniades MA, Eleftheriades GV (2010) A compact triband monopole antenna with single-cell metamaterial loading. IEEE Trans Antennas Propag 58:1031-1038. https://doi. org/10.1109/TAP.2010.204131

21. Ntaikos DK, Bourgis NK, Yioultsis TV (2011) Metamaterial -based electrically small multiband planar monopole antennas. IEEE Antennas Wirel Propag Lett 10:963-966. https://doi. org/10.1109/LAWP.2011.2167309

22. Islam M, Islam MT, Samsuzzaman M, Faruque MRI (2015) A negative index metamaterial antenna for UWB microwave imaging applications. Microw Opt Technol Lett 57:1352-1361. https://doi.org/10.1002/mop.29095

23. Murugan HA, Gudibandi BR, Damodharan SK (2019) Circularly polarized dual-band zeroth order epsilon negative antenna using asymmetric unit cells. Int J RF Microw Comput Aided Eng. https://doi.org/10.1002/mmce.21827

24. Marathe D, Kulat K (2017) A compact triple-band negative permittivity metamaterial for C, X-band applications. Int J Antennas Propag 2017:1-12. https://doi.org/10.1155/2017/7515264

25. Hossain M, Faruque M, Islam M, Ullah M (2015) A new wideband double-negative metamaterial for C-and S-band applications. Materials 8:57-71. https://doi.org/10.3390/ma801 0057

26. Hasan M, Faruque M, Islam S, Islam M (2016) A new compact double-negative miniaturized metamaterial for wideband operation. Materials 9:830. https://doi.org/10.3390/ma9100830

27. Hossain MJ, Faruque MR, Alam M, Mansor MF, Islam MT (2018) A broadband negative refractive index meta-atom for quad-band and sensor applications. Microw Opt Technol Lett 60:2899-2907. https://doi.org/10.1002/mop.31410

28. Islam S, Hasan M, Faruque MRI, Islam MT (2017) Two components NRI metamaterial for dual band applications. Microw Opt Technol Lett 59:1092-1096. https://doi.org/10.1002/mop.30468

29. Alam MJ, Faruque MRI, Azim R, Islam MT (2018) Depiction and analysis of a modified $\mathrm{H}$-shaped double-negative meta atom for satellite communication. Int J Microw Wireless Technol 10:1155-1165. https://doi.org/10.1017/S1759078718001022

30. Islam S, Faruque M, Islam M (2014) The design and analysis of a novel split-H-shaped metamaterial for multi-band microwave applications. Materials 7:4994-5011. https://doi.org/10.3390/ ma7074994
31. Schurig D, Mock J, Smith D (2006) Electric-field-coupled resonators for negative permittivity metamaterials. Appl Phys Lett 88:041109. https://doi.org/10.1063/1.2166681

32. Withayachumnankul W, Fumeaux C, Abbott D (2010) Compact electric-LC resonators for metamaterials. Opt Express 18:2591225921. https://doi.org/10.1364/OE.18.025912

33. Kolb P, Salter T, McGee J, Drew H, Padilla W (2011) Extreme subwavelength electric GHz metamaterials. J Appl Phys 110:054906 (1-5). https://doi.org/10.1063/1.3633213

34. Dhouibi A, Burokur SN, de Lustrac A, Priou A (2012) Z-shaped meta-atom for negative permittivity metamaterials. Appl Phys A 106:47-51. https://doi.org/10.1007/s00339-011-6673-9

35. Dhouibi A, Burokur SN, de Lustrac A, Priou A (2012) Comparison of compact electric-LC resonators for negative permittivity metamaterials. Microw Opt Technol Lett 54:2287-2295. https:// doi.org/10.1002/mop. 27050

36. Sarkhel A, Mitra D, Chaudhuri SRB (2016) A compact metamaterial with multi-band negative-index characteristics. Appl Phys $A$ 122:471. https://doi.org/10.1007/s00339-016-0005-z

37. Sarkhel A, Mitra D, Paul S, Chaudhuri SRB (2015) A compact meta-atom for dual band negative permittivity metamaterial. Microw Opt Technol Lett 57:1152-1156. https://doi.org/10.1002/ mop.29041

38. Zarghooni B, Dadgarpour A, Denidni TA (2015) Greek-key pattern as a miniaturized multiband metamaterial unit-cell. IEEE Antennas Wirel Propag Lett 14:1254-1257. https://doi. org/10.1109/LAWP.2015.2400820

39. Li B, He L, Yin Y, Guo W, Sun X (2013) An isotropy dual-band terahertz metamaterial. Microw Opt Technol Lett 55:988-990. https ://doi.org/10.1002/mop.27486

40. Sabah C (2010) Novel, dual band, single and DNG metamaterials: nonconcentric delta loop resonators. Prog Electromagn Res 25:225-239. https://doi.org/10.2528/PIERB10080302

41. Du G, Liu C (2012) Multiband metamaterial structure: Butterflypattern resonator. Microw Opt Technol Lett 54:2179-2181. https ://doi.org/10.1002/mop.27027

42. Turkmen O, Ekmekci E, Turhan-Sayan G (2012) Nested U-ring resonators: a novel multi-band metamaterial design in microwave region. IET Microw Antennas Propag 6:1102-1108. https ://doi.org/10.1049/iet-map.2012.0037

43. Islam S, Faruque M, Islam M (2015) A near zero refractive index metamaterial for electromagnetic invisibility cloaking operation. Materials 8:4790-4804. https://doi.org/10.3390/ma808 4790

44. Smith K, Adams RS (2018) A broadband negative epsilon fractal metamaterial unit cell for coaxial notch filter applications. Prog Electromagn Res 86:257-267. https://doi.org/10.2528/PIERC 18030204

45. Razak A, Rahim M, Majid H, Murad N (2017) Frequency reconfigurable epsilon negative metamaterial antenna. Int J Electr Comput Eng 7:1473-1479. https://doi.org/10.11591/ijece.v7i3

46. Li K, Zhu C, Li L, Cai Y-M, Liang C-H (2013) Design of electrically small metamaterial antenna with ELC and EBG loading. IEEE Antennas Wirel Propag Lett 12:678-681. https://doi. org/10.1109/LAWP.2013.2264099

47. Dalgaç Ş, Karadağ F, Ünal E, Özkaner V, Bakır M, Akgöl O, Sevim UK, Delihacıoğlu K, Öztürk M, Karaaslan M (2020) Metamaterial sensor application concrete material reinforced with carbon steel fiber. Mod Phys Lett B. https://doi.org/10.1142/S021798492 0500979

48. Tamer A, Karadağ F, Ünal E, Abdulkarim YI, Deng L, Altintas O, Bakır M, Karaaslan M (2020) Metamaterial based sensor integrating transmission line for detection of branded and unbranded diesel fuel. Chem Phys Lett 742:137169. https://doi. org/10.1016/j.cplett.2020.137169 
49. Abdulkarim $\mathrm{Yl}$, Deng L, Karaaslan M, Altıntaş O, Awl HN, Muhammadsharif FF, Liao C, Unal E, Luo H (2020) Novel metamaterialsbased hypersensitized liquid sensor integrating omega-shaped resonator with microstrip transmission line. Sensors 20(3):943. https://doi.org/10.3390/s20030943

50. Landy NI, Sajuyigbe S, Mock JJ, Smith DR, Padilla WJ (2008) Perfect metamaterial absorber. Phys Rev Lett 100(20):207402. https ://doi.org/10.1103/PhysRevLett.100.207402

51. Nicolson A, Ross G (1970) Measurement of the intrinsic properties of materials by time-domain techniques. IEEE Trans Instrum Meas 19:377-382

52. Barroso J, De Paula A (2010) Retrieval of permittivity and permeability of homogeneous materials from scattering parameters.
J Electromagn Waves Appl 24(11-12):1563-1574. https://doi. org/10.1163/156939310792149759

53. Bahl IJ, Bhartia P (2003) Microwave solid state circuit design. Wiley, Hoboken

54. Gevorgian S, Berg H (2001) Line capacitance and impedance of coplanar-strip waveguides on substrates with multiple dielectric layers.

Publisher's Note Springer Nature remains neutral with regard to jurisdictional claims in published maps and institutional affiliations. 